DOI: $10.15593 / 2224-9982 / 2016.47 .12$

УДК 532

\author{
Д.Ф. Гайнутдинова
}

Пермский национальный исследовательский

политехнический университет, Пермь, Россия

\author{
РАЗРАБОТКА МЕТОДИКИ ПРОВЕДЕНИЯ \\ ФИЗИЧЕСКИХ ЭКСПЕРИМЕНТОВ ПО ИССЛЕДОВАНИЮ \\ ВЗАИМОВЛИЯНИЯ В ДИНАМИЧЕСКОЙ СИСТЕМЕ \\ жИДКОсть - кОНстРукцИЯ
}

\begin{abstract}
В настоящее время широкое распространение получили гидравлические системы, включающие высокооборотные шнеко-центробежные насосы, которые обычно работают в условиях вибраций элементов их конструкции. Возникающие при этом вынужденные колебания давлений жидкости могут приводить к различным нежелательным и опасным явлениям, таким как кавитационный срыв работы насоса, неравномерная подача жидкости, потеря устойчивости и др. Эта проблема актуальна и недостаточно изучена. В данной работе выдвигается предположение о влиянии вибраций элементов конструкции насоса на кавитацию. Разработана модельная экспериментальная установка «Гидроупругость» для исследования быстропротекающих гидродинамических процессов с учетом взаимовлияния жидкости, газа и деформируемой конструкции. Описана методика проведения фризических экспериментов на разработанной установке. Проведен физический эксперимент. В ходе анализа проведенных физических экспериментов исследованы кавитационные эффекты при вибрациях. Обнаружено усиление кавитационных эффектов на частотах, близких к резонансным для данной системы. На основе полученных результатов можно предположить, что непрогнозируемые опасные явления, типа кавитационного срыва насоса, могут усиливаться гидрогазодинамическими процессами, которые зависят от вибраций элементов конструкции насоса.

Ключевые слова: гидроупругость, физический эксперимент, методика, экспериментальная установка, кавитационный срыв, вибрации, гидродинамические процессы.
\end{abstract}

\author{
D.F. Gaynutdinova
}

Perm National Research Politechnic University, Perm, Russian Federation

\title{
DEVELOPMENT OF TECHNIQUE OF PHYSICAL EXPERIMENTS TO STUDY INTERACTION IN THE DYNAMIC SYSTEM LIQUID - DESIGN
}

At present, widely used hydraulic system comprises a high speed auger-centrifugal pumps, which are usually operated under vibration elements of their design. Emerging with the forced oscillations of fluid pressure can lead to a variety of unwelcome and dangerous phenomena, such as the 
failure of the pump cavitation, uneven supply of liquid, buckling, etc. This is a problem, and poorly understood. In this paper we put forward the assumption of influence of vibration of the pump cavitation design elements. A model experimental setup "Hydroelasticity" for studying rapid hydrodynamic processes taking into account the mutual influence of the liquid, gas and the deformable structure. The technique of physical experiments on the developed installation. Spend a physical experiment. The analysis conducted physical experiments investigated the effects of cavitation during the vibrations. Detected gain cavitation effects at frequencies close to the resonance of the system. It can be assumed that the unpredictable hazards, such as pump cavitation breakdown, may increase hydro-processes that depend on the vibration of the pump design elements on the basis of the results obtained.

Keywords: hydroelasticity, physical experiment, technique, experimental setup, breakdown cavitation, vibration, hydrodynamic processes.

\section{Введение}

Проблема непрогнозируемого возникновения кавитации в насосах является актуальной и изучается на протяжении многих лет. В частности, этой проблеме посвящены работы таких известных авторов, как Т.М. Башта, А.М. Матвеенко, М.С. Натанзон, В.Г. Кинелев, А.И. Гулиенко, В.М. Калнин, В.А. Шерстянников, С.И. Перевощиков, М.А. Промтов и др. ${ }^{1}[1-12]$. Авторы занимаются исследованием кавитационных процессов в насосах, исследуют влияние кавитации на вибрацию $[5,6]$. Однако проблема далека от разрешения, в частности, недостаточно работ по исследованию влияния вибраций на кавитацию. Предположительно вибрации могут приводить к кавитации, которая вызывает срыв напора, износ и разрушение элементов конструкции. В данной работе экспериментально исследуется возникновение кавитации при действии вибрации. Разработана экспериментальная установка по исследованию этих процессов и проведены физические эксперименты.

\section{Экспериментальная установка «Гидроупругость»}

Экспериментальная установка «Гидроупругость» создана для исследования влияния вибраций на кавитационные эффекты с учетом взаимовлияния жидкости, газа и деформируемой конструкции (рис. 1) [13-16]. Кроме того, установка позволит верифицировать полученные на суперкомпьютере результаты численного моделирования этих явлений [17-19].

Установка представляет собой систему, состоящую из трех основных узлов: модельная рабочая камера, узел нагружения и измери-

\footnotetext{
${ }^{1}$ Чаплыгин В.Я., Матвеенко А.М. Способ создания высоких и сверхвысоких давлений и устройство для его осуществления: пат. № 2502894. 2012.
} 
тельно-задающий вычислительный комплекс (ИЗВК). Модельная камера представляет собой трубу с фланцами для установки на стапеле (основании) и крепления дополнительного оборудования и фитингами для установки гидрофонов. В качестве узла нагружения выступает пьезокерамический излучатель и устройство усиления и модуляции входного сигнала. ИЗВК состоит из трех датчиков давления (гидрофонов); трех датчиков виброускорений РСВ 352C03; модуля согласования сигналов; шасси National Instruments (NI) PXI 1050 с установленным 8-канальным модулем NI PXI 4472B измерения динамических сигналов и программным обеспечением LabVIEW.11.

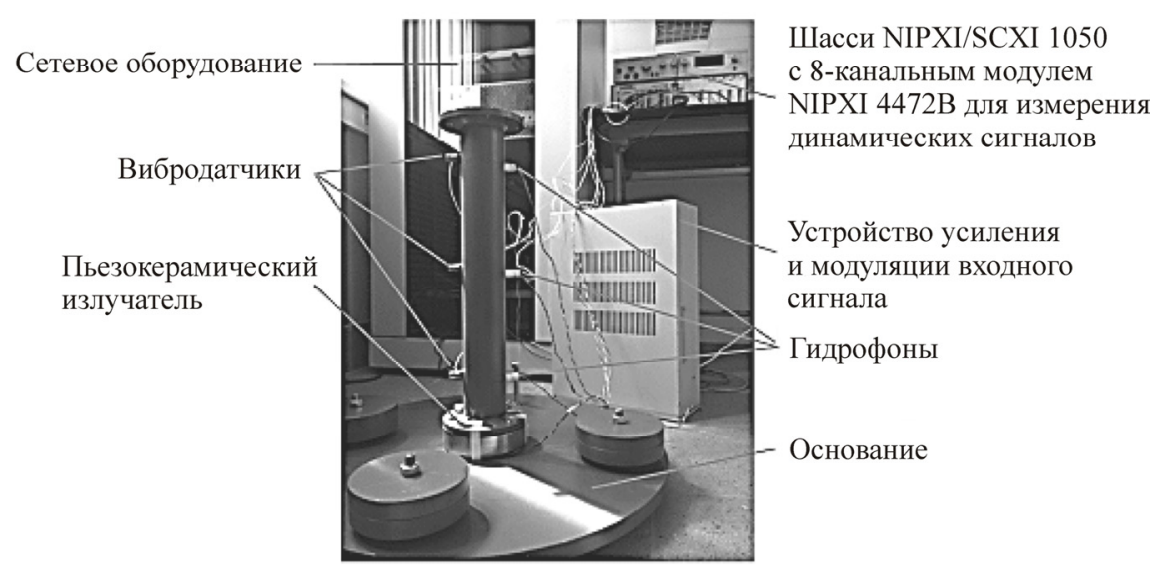

Рис. 1. Экспериментальная установка «Гидроупругость»

Для возбуждения колебаний на узел нагружения подаются управляющие сигналы, формирующиеся в программном комплексе LabVIEW.11.

\section{Методика проведения физических экспериментов}

Сформулируем основные этапы физического эксперимента:

1. Подготовка физического эксперимента

В качестве рабочего тела могут использоваться различные жидкости (вода, спирты, нефтепродукты и т.д.). В настоящем исследовании использовалась вода. При проведении исследований влияния характеристик рабочего тела на вибрационные и кавитационные характеристики процесса перед каждым экспериментом проводится:

- очистка внутренней поверхности рабочей камеры; 
- визуальный осмотр рабочей камеры, приборов, проводов, узлов соединений;

- фиксирование условий проведения экспериментов (температура, давление окружающей среды);

- тарировка датчиков (в случае их замены).

2. Запуск и настройка устройства усиления и модуляции сигнала

Нелинейные акустические колебания формируются путем создания в воде ультразвуковых колебаний высокой (более 1 Вт/см²) интенсивности, а линейные акустические колебания формируют путем амплитудной модуляции используемых ультразвуковых колебаний ${ }^{2}$. Ультразвуковая составляющая механическим путем отфильтровывается вблизи излучателя.

3. Запуск и настройка шасси National Instrument PXI 1050

Регистрация и предварительный анализ быстропротекающих процессов в рабочей камере выполняются с использованием программно-аппаратного комплекса на базе National Instruments NI PXI1050 (рис. 2). Измерительная система создана на базе шасси NIPXIISCXI 1050 с установленным модулем сбора динамических сигналов NIPXI 4472 B.

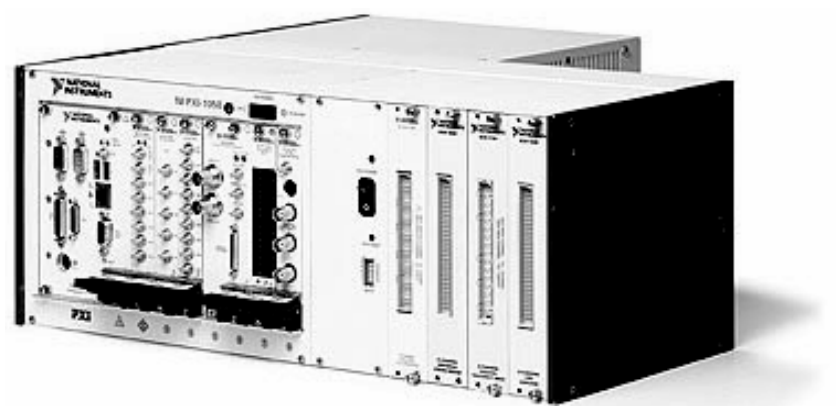

Рис. 2. National Instruments NI PXI-1050

4. Запуск и настройка виртуального прибора, созданного на базе программного комплекса LabVIEW.11

Для проведения физических экспериментов на экспериментальной установке используется прикладная программа «Гидроупругость»,

\footnotetext{
${ }^{2}$ На устройство усиления и модуляции сигнала подана заявка на патент на изобретение «Способ проведения эксперимента по осуществлению и наблюдению акустических процессов в жидкой среде и устройство для его осуществления». Приоритет № 2015139637 от 17.09.2015.
} 
разработанная в среде LabVIEW.11, - виртуальный прибор (ВП). Компонентами ВП являются передняя панель, блок-диаграмма и пиктограмма/коннектор. Передняя панель реализует пользовательский интерфейс с ВП, позволяет задавать исходные данные и отображать результаты работы ВП. Блок-диаграмма является аналогом традиционной программы и реализует функциональные возможности ВП. Пиктограмма/коннектор позволяют использовать ВП в качестве подпрограммы (SubVI, виртуальный «подприбор») при построении модульных иерархических программ.

При запуске программы открывается рабочее окно - интерактивный интерфейс пользователя. На рис. 3 показано рабочее окно программы «Гидроупругость», где отображаются параметры, необходимые для работы экспериментальной установки.

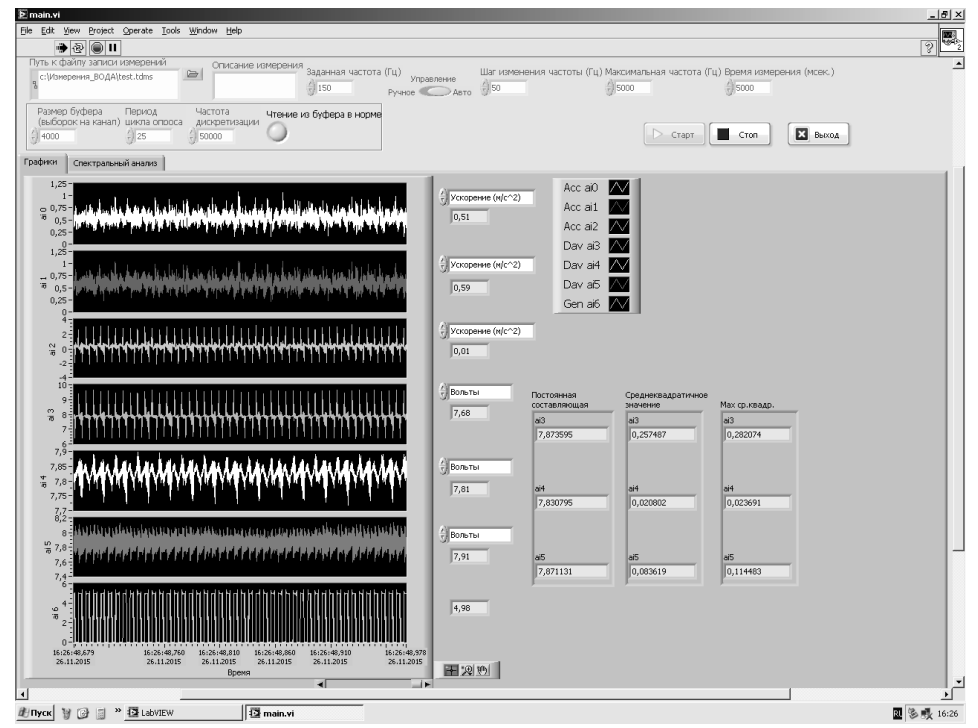

Рис. 3. Рабочее окно виртуального прибора LabVIEW.11

В верхней части рабочего окна виртуального прибора в соответствии с планом физических экспериментов задается частота считывания данных из буфера, частота дискретизации, требуемая частота нагружения, диапазон изменения параметров и шаг измерений.

На рабочее поле выводятся семь графиков (см. рис. 3), которые отображают результаты эксперимента, с накоплением. По горизонтальной оси откладываются номера выборок. Три верхних графика 
(ai0, ai1, ai2) отражают данные, получаемые с вибродатчиков; следующие три графика (ai3, ai4, ai5) - данные, получаемые с гидрофонов; нижний график (ai6) соответствует сигналу генератора, подаваемому на узел нагружения.

\section{5. Проведение серий физических экспериментов}

В соответствии с планом проводим серию физических экспериментов, в которых варьируются частота и амплитуда колебаний в автоматическом или ручном режимах.

6. Обработка, отображение и сохранение результатов экспериментов

Для того чтобы просмотреть сохраненные файлы, необходимо открыть один из модулей программы LabeVIEW для отображения результатов экспериментов - TDMS - FileViewer.vi. Данные отображаются на трех вкладках: Properties - свойства, Values (table) - значение параметра в табличном виде, Analogvalues (graph) - значения параметра в графическом виде (рис. 4).

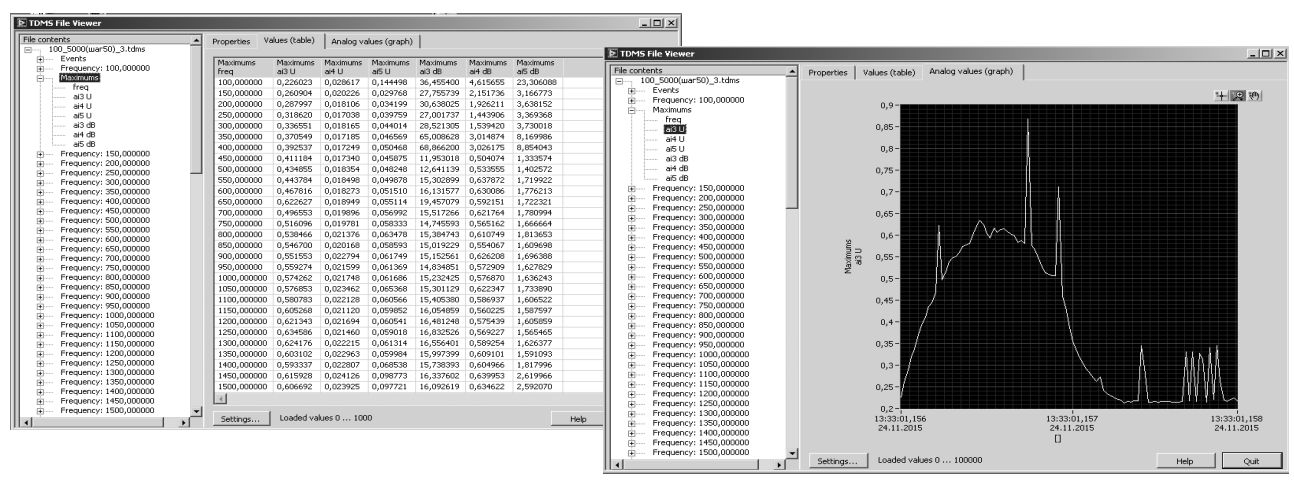

Рис. 4. Поле отображения результатов эксперимента в LabeVIEW.11

Для проведения дальнейшего анализа необходимо экспериментальные данные экспортировать в табличном виде в Microsoft Excel, а полученные графики сохранять в виде изображений.

\section{Результаты проведения физических экспериментов}

В ходе физических экспериментов узел нагружения, расположенный на нижнем торце гидродинамической полости модельной камеры, совершал колебания с заданной частотой. Частоты нагружения варьи- 
ровались от 0 до 5 кГц. Длина рабочей камеры - от 0,4 до 1,0 м. Столб жидкости - от 0,4 до 0,7 м. При этом фиксировались изменения давления жидкости во времени. Получены зависимости амплитуд вынужденных колебаний системы от частоты нагружения при различных геометрических размерах рабочей камеры, высотах столба жидкости, массовых характеристиках конструкции.

На рис. 5 показаны зависимости максимумов среднеквадратичных значений амплитуды от частоты. Из графика видно, что на частоте, приблизительно равной 1500 Гц, отчетливо наблюдается усиление сигналов. Видно, что система откликается на собственную парциальную частоту жидкости, которая составляет, по результатам аналитического расчета, 1700 Гц. На рис. 5 виден всплеск на частоте генератора 2500 Гц. Это означает, что система откликается на собственную парциальную частоту конструкции, которая составляет, по результатам модального анализа в ANSYS, 2470 Гц.

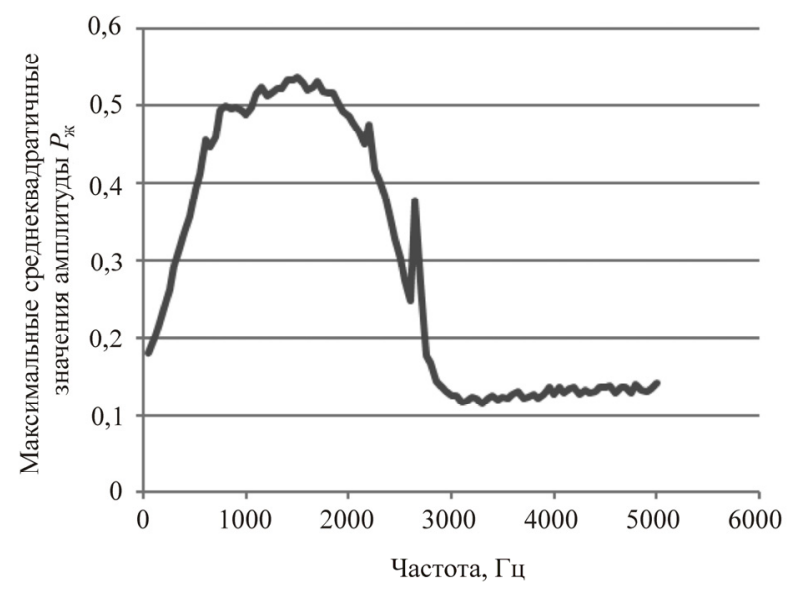

Рис. 5. Зависимость максимумов среднеквадратичных значений амплитуды колебаний давления от частоты

На рис. 6 показаны зависимости максимумов среднеквадратичных значений амплитуды от частоты при одинаковой массе присоединенного груза ( $m=3,2$ кг) и длине дополнительного канала $\left(h_{\mathrm{\kappa}}=0\right.$ м, $h_{\mathrm{\kappa}}=0,66$ м). Анализ измеренных сигналов показал, что изменение длины канала в заданном диапазоне влияет на характеристики колебаний рабочего тела незначительно, в пределах $10 \%$. Это объясняется тем, что верхний торец столба жидкости - свободная поверхность, которая 
не соприкасается с конструкцией. Таким образом, удлинение верхней части рабочей камеры не оказывает существенного влияния на колебания жидкости.

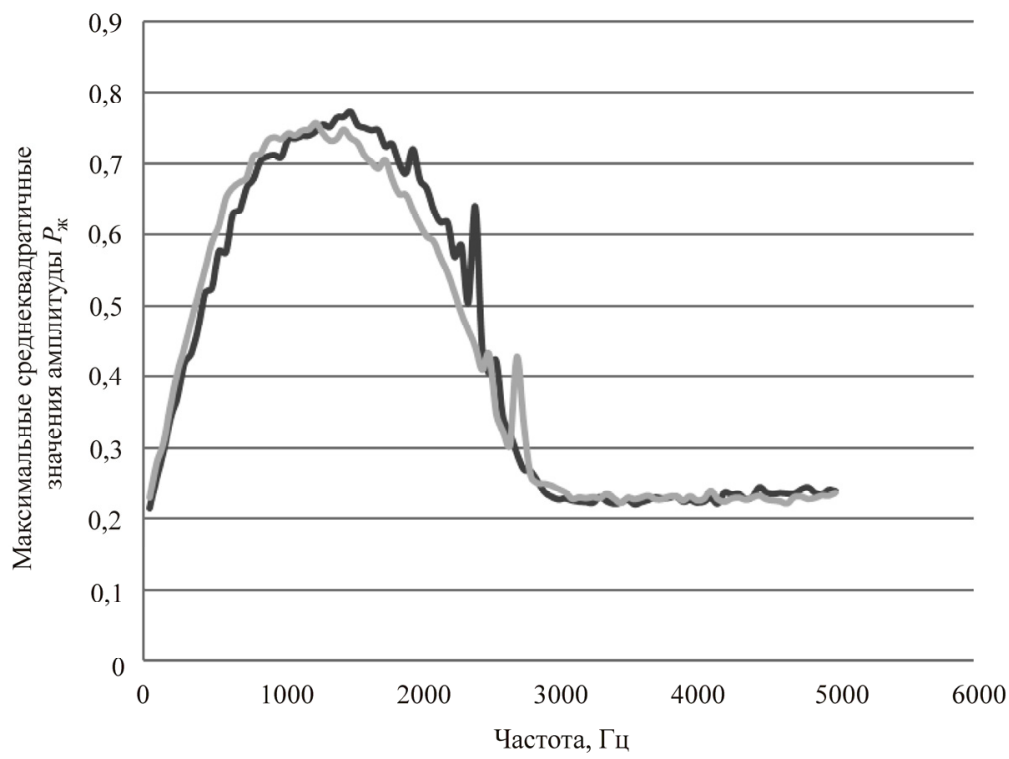

Рис. 6. Зависимость максимумов среднеквадратичных значений амплитуды колебаний давления от частоты при $m=3,2$ кг,

$$
h_{\text {ж }}=0,44 \mathrm{м}:--h_{\mathrm{\kappa}}=0 \mathrm{M},--h_{\mathrm{\kappa}}=0,66 \mathrm{м}
$$

На рис. 7 показаны зависимости максимумов среднеквадратичных значений амплитуды от частоты при различных высотах столба жидкости. При сравнении двух расчетных вариантов видно, что с увеличением высоты столба жидкости величина максимумов среднеквадратичных значений амплитуды сигналов увеличивается приблизительно в два раза. Это связано с тем, что при увеличении высоты столба жидкости наблюдается увеличение объема кавитационных пузырьков в резонансной области. В рассматриваемом случае объем увеличился в 1,68 раз.

На фотографиях показаны результаты физических экспериментов (рис. 8). Съемка производилась сверху со стороны открытой части рабочей камеры. Визуально обнаружено, что на частотах свыше 800 Гц возникают кавитационные пузырьки. Наиболее интенсивная кавитация наблюдается в диапазоне частот от 800 до 3000 Гц. Это объясняется близостью к резонансной для столба жидкости частоте, равной 1700 Гц. 
При повышении частоты выше 3000 Гц кавитация наблюдается, но с меньшей интенсивностью, что и видно на графиках рис. $6,7$.

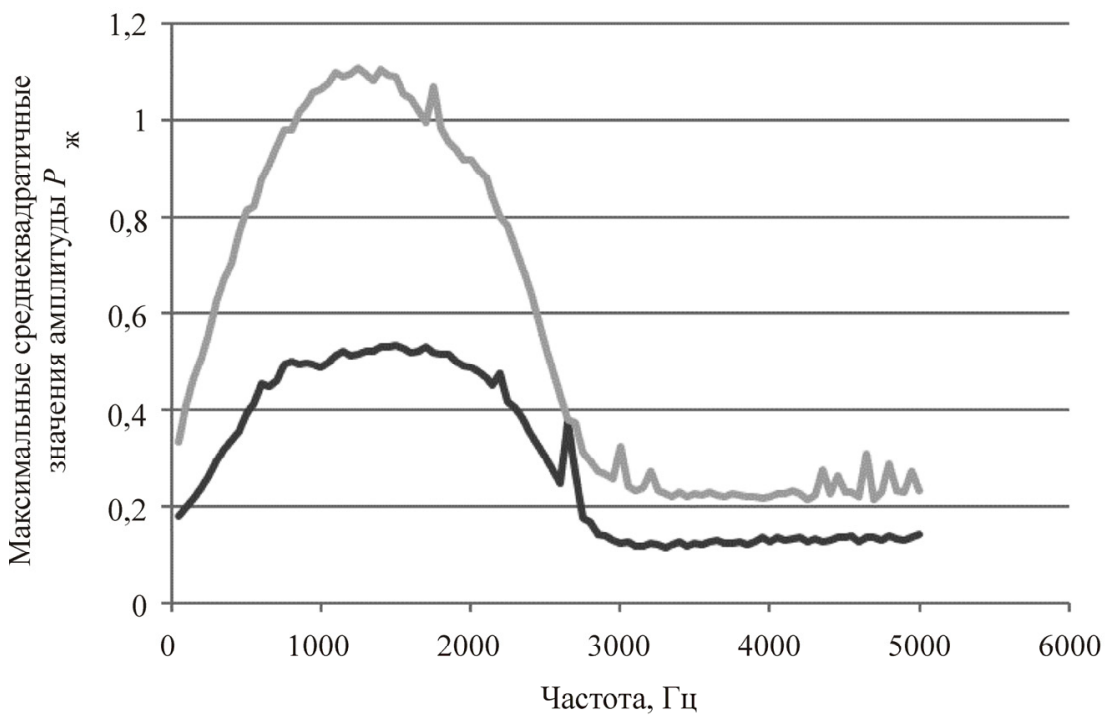

Рис. 7. Зависимость максимумов среднеквадратичных значений амплитуды колебаний давления от частоты при различных $h_{\text {ж: }}--0,44$ м; $--0,74$ м

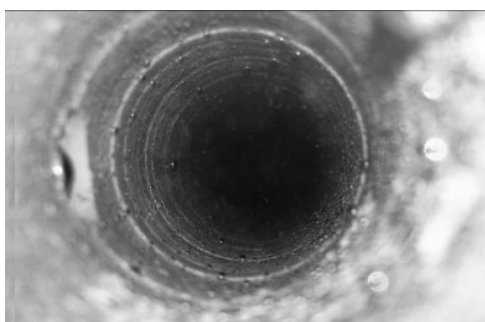

$a$

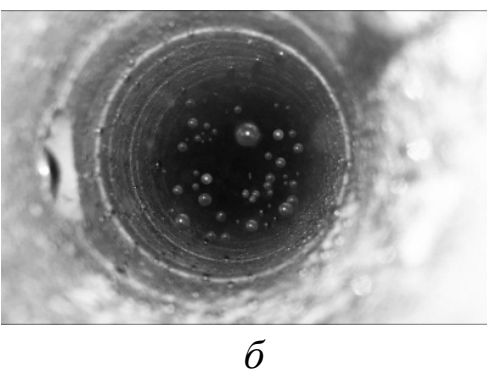

$\sigma$

Рис. 8. Результаты физических экспериментов: $a-f=100$ Гц - нет кавитации; $\sigma-f=1500$ Гц - наблюдаются кавитационные пузырьки

Совместный анализ графических зависимостей и фотографий процесса показывает, что имеет место пороговое среднеквадратичное значение амплитуды колебаний давления, при котором наблюдается кавитация. Оно принимает значение в диапазоне $0,3-1,0$ (см. рис. 8). На рис. 7 диапазон изменения порогового среднеквадратичного значе-

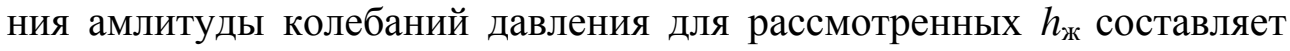


0,5-0,9, при этом диапазон приведенных (к объему жидкости) пороговых значений значительно у́же и составляет 1852-1978. Введение приведенного порогового значения позволит оценивать условия возникновения кавитации в каналах различной геометрии.

\section{Заключение}

1. В ходе физических экспериментов подтверждено и исследовано возникновение кавитационных эффектов при вибрациях.

2. Обнаружено усиление кавитационных эффектов на частотах нагружения, близких к собственной продольной частоте жидкостного столба.

3. Обнаружено усиление кавитационных эффектов на частотах, близких к резонансным для данной конструкции.

4. Совместный анализ графических зависимостей и фотографий процесса показал, что имеет место пороговое среднеквадратичное значение амплитуды колебаний давления, при котором наблюдается кавитация. Оно принимает значение в диапазоне 0,3-1,0. При этом приведенное пороговое среднеквадратичное значение амплитуды колебаний давления изменяется в диапазоне от 1852 до 1978, что удобно для оценки условий возникновения кавитации в каналах различной геометрии.

5. На основе полученных результатов можно предположить, что непрогнозируемое снижение напора насоса может быть вызвано гидрогазодинамическими процессами, возникающими при вибрациях элементов конструкции.

Работа выполнена при финансовой поддержке гранта Российского научного фонда (проект № 14-19-00877).

\section{Библиографический список}

1. Гулиенко А.И. Уравнения движения жидкости в вибрирующих трубопроводах гидросистем // Математические модели рабочих процессов в гидропневмосистемах: сб. науч. тр. - Киев: Наук. думка, 1981. C. 111-122.

2. Гулиенко А.И., Калнин В.М., Шерстянников В.А. Вынужденные колебания давления жидкости в гидросистеме при вибрациях // Тр. ЦИАМ. - 1980. - № 895. - С. 229-234. 
3. Карелин В.Я. Кавитационные явления в центробежных и осевых насосах. - 2-е изд. - М.: Машиностроение, 1975. - 335 с.

4. Пилипенко В.В., Задонцев В.А., Натанзон М.С. Кавитационные автоколебания и динамика гидросистем. - М.: Машиностроение, 1977. - $352 \mathrm{c}$.

5. Перевощиков С.И., Безус А.А. Вибрация насосно-силовых агрегатов магистральных нефтепроводов // Нефтяное хозяйство. - 1994. № 10. - C. 54-55.

6. Перевощиков С.И. Снижение гидродинамической вибрации центробежных насосов // Известия вузов. Нефть и газ. - 1997. - № 4. C. 50-56.

7. Чаплыгин В.Я., Матвеенко А.М. Исследование течения жидкости в несимметричных гидравлических каналах // Вестник Моск. авиац. ин-та. - 2012. - Т. 19, № 1. - С. 58-64.

8. Чаплыгин В.Я., Матвеенко А.М. Кавитация в зазорах с учетом вязкости жидкости и ускорения элементов конструкций // Динамические и технологические проблемы механики конструкций и сплошных сред: материалы XX Междунар. симпоз. им. А.Г. Горшкова. - М., 2014. - C. 142-144.

9. Злоткин Ш.Л. Движение жидкости в вибрирующем трубопроводе // Труды ВВИА им. Н.Е. Жуковского. - 1948. - Вып. 311.

10. Xiu-Tian Y., Guoyuan, Z., Analysis of two phase flow in liquid oxygen hybrid journal bearings for rocket engine turbopumps // Industrial Lubrication and Tribology. - 2014. - Vol. 66, № 1. - P. 31-37.

11. Experimental and computational investigation of the nasa lowspeed centrifugal compressor flow field / J.R. Wood, A.J. Strazisar, M.D. Hathaway, R.M. Chriss // AMSE Journal of Turbomachinery. 1993. - Vol. 115. - P. 527-542.

12. Ismaier A., Schlcker E. Fluid dynamic interaction between water hammer and centrifugal pumps // Nuclear Engineering and Design. 2009. - Vol. 239. - P. 3151-3154.

13. Гайнутдинова Д.Ф., Модорский В.Я., Масич Г.Ф. Проектирование технологической платформы для экспериментальных и вычислительных исследований быстропротекающих процессов гидроупругости // Науч.-техн. вестник Поволжья. - 2014. - № 5. - С. 155-158.

14. Gaynutdinova D.F., Modorsky V.Ya., Masich G.F. Infrastructure of data distributed processing in high-speed process research based on 
hydroelasticity task // Procedia Computer Science. - 2015. - Vol. 66. P. 556-563.

15. The development of the technology platform on the basis of a supercomputer to handle large flows of experimental data / D.F. Gaynutdinova, V.Ya. Modorsky, V.A. Shchapov, D.P. Chugunov, G.F. Masich // CEUR Workshop Proceedings: Online Proc. for Sci. Workshops. Parallel Computing Technologies (PCT'2016). Arkhangelsk, March 29-31. Arkhangelsk, 2016. - Vol. 1576. - P. 490-498.

16. Optimization in design of scientific products for purposes of cavitation problems / V.Ya. Modorskii, D.F. Gaynutdinova, V.P. Gergel, K.A. Barkalov // AIP Conference Proceedings. - 2016. - 1738. - 400013. DOI: org/10.1063/1.4952201

17. Гайнутдинова Д.Ф., Модорский В.Я., Козлова А.В. Вычислительное моделирование области возникновения кавитации при вибрациях // Науч.-техн. вестник Поволжья. - 2014. - № 6. - С. 127-129.

18. Решение инженерных задач на высокопроизводительном вычислительном комплексе Пермского национального исследовательского политехнического университета: моногр. / под ред. В.Я. Модорского. - Пермь: Изд-во Перм. нац. исслед. политехн. ун-та, 2014. - 314 с.

19. Модорский В.Я., Соколкин Ю.В. Газоупругие процессы в энергетических установках. - М.: Наука, 2007.- 176 с.

\section{References}

1. Gulienko A.I. Uravneniya dvizheniya zhidkosti v vibriruyushchikh truboprovodakh gidrosistem [The equations of fluid motion in the vibrating pipelines of hydraulic systems]. Matematicheskie modeli rabochikh protsessov v gidropnevmosistemakh. Kiev: Naukova dumka, 1981, pp. 111-122.

2. Gulienko A.I., Kalnin V.M., Sherstyannikov V.A. Vynuzhdennye kolebaniya davleniya zhidkosti $\mathrm{v}$ gidrosisteme pri vibratsiyakh [Forced oscillations of fluid pressure in the hydraulic system under vibrations]. Trudy Tsentralnogo instituta aviatsionnogo motorostroeniya, 1980, no. 895. pp. 229-234.

3. Karelin V.Ya. Kavitatsionnye yavleniya $v$ tsentrobezhnykh i osevykh nasosakh [Cavitation phenomena in centrifugal and axial pumps]. Moscow: Mashinostroenie, 1975. 335 p. 
4. Pilipenko V.V., Zadontsev V.A., Natanzon M.S. Kavitatsionnye avtokolebaniya i dinamika gidrosistem [Cavitational self-oscillations and dynamics of hydraulic systems]. Moscow: Mashinostroenie, 1977. 352 p.

5. Perevoshchikov S.I., Bezus A.A. Vibratsiya nasosno-silovykh agregatov magistralnykh nefteprovodov [Vibration of pump and power units of trunk oil pipelines]. Neftyanoe khozyaystvo, 1994, no. 10, pp. 54-55.

6. Perevoshchikov S.I. Snizhenie gidrodinamicheskoy vibratsii tsentrobezhnykh nasosov [Reducing hydrodynamic vibration of centrifugal pumps]. Izvestia vusshykh uchebnykh zavedeniy. Neft i gaz, 1997, no. 4, pp. 50-56.

7. Chaplygin V.Ya., Matveenko A.M. Issledovanie techeniya zhidkosti $\mathrm{v}$ nesimmetrichnykh gidravlicheskikh kanalakh [The research of fluid motion in asymmetric hydraulic channels]. Vestnik Moskovskogo aviatsionnogo instituta, 2012, vol. 19, no. 1, pp. 58-64.

8. Chaplygin V.Ya., Matveenko A.M. Kavitatsiya v zazorakh s uchetom vyazkosti zhidkosti i uskoreniya elementov konstruktsiy [Cavitation in the gaps considering liquid viscosity and the acceleration of structural elements.]. Materialy XX Mezhdunarodnogo simpoziuma "Dinamicheskie $i$ tekhnologicheskie problemy mekhaniki konstruktsiy i sploshnykh sred" imeni A.G. Gorshkova. Moscow, 2014, pp. 142-144.

9. Zlotkin Sh.L. Dvizhenie zhidkosti v vibriruyushchem truboprovode [Fluid motion in a vibrating pipeline]. Trudy Voenno-vozdushnoy inzhenernoy akademii imeni N.E. Zhukovskogo, 1948, iss. 311.

10. Xiu-Tian Y., Guoyuan Z. Analysis of two phase flow in liquid oxygen hybrid journal bearings for rocket engine turbopumps. Industrial Lubrication and Tribology, 2014, vol. 66, no. 1, pp. 31-37.

11. Wood J.R., Strazisar A.J., Hathaway M.D., Chriss R.M. Experimental and computational investigation of the NASA low-speed centrifugal compressor flow field. AMSE Journal of Turbomachinery, 1993, vol. 115, pp. 527-542.

12. Ismaier A., Schlcker E. Fluid dynamic interaction between water hammer and centrifugal pumps. Nuclear Engineering and Design, 2009, vol. 239, no. 12, pp. 3151-3154.

13. Gaynutdinova D.F., Modorskiy V.Ya., Masich G.F. Proektirovanie tekhnologicheskoy platformy dlya eksperimentalnykh i vychislitelnykh issledovaniy bystroprotekayushchikh protsessov gidrouprugosti [Design of the technological platform for experimental and computing research of the 
fast hydroelastic processes]. Nauchno-tekhnicheskiy vestnik Povolzhya, 2014, no. 5, pp. 155-158.

14. Gaynutdinova D.F., Modorsky V.Ya., Masich G.F. Infrastructure of data distributed processing in high-speed process research based on hydroelasticity task. Procedia Computer Science, 2015, vol. 66, pp. 556-563.

15. Gaynutdinova D.F., Modorsky V.Ya., Shchapov V.A., Chugunov D.P., Masich G.F. The development of the technology platform on the basis of a supercomputer to handle large flows of experimental data. CEUR Workshop Proceedings. Online Proc. for Sci. Workshops. Parallel Computing Technologies (PCT'2016), 2016, vol. 1576, pp. 490-498.

16. Modorskii V.Ya., Gaynutdinova D.F., Gergel V.P., Barkalov K.A. Optimization in design of scientific products for purposes of cavitation problems. AIP Conference Proceedings, 1738, 400013 (2016). DOI: org/10.1063/1.4952201

17. Gaynutdinova D.F., Modorskiy V.Ya., Kozlova A.V. Vychislitelnoe modelirovanie oblasti vozniknoveniya kavitatsii pri vibratsiyakh [Computing modeling of area of emergence of cavitation under vibration]. Nauchno-tekhnicheskiy vestnik Povolzhya, 2014, no. 6, pp. 127-129.

18. Reshenie inzhenernykh zadach na vysokoproizvoditelnom vychislitel'nom komplekse Permskogo natsionalnogo issledovatelskogo politekhnicheskogo universiteta [The solution of engineering problems on a high performance-computing complex of the Perm National Research Polytechnic University]. Ed. by V.Ya. Modorskiy. Permskiy natsionalnyy issledovatelskiy politekhnicheskiy universitet, 2014. 314 p.

19. Modorskiy V.Ya., Sokolkin Yu.V. Gazouprugie protsessy v energeticheskikh ustanovkakh [Gas elastic processes in power plants]. Moscow: Nauka, 2007. 176 p.

\section{Об авторе}

Гайнутдинова Динара Фирдаусовна (Пермь, Россия) - аспирант кафедры «Механика композиционных материалов и конструкций», инженер Центра высокопроизводительных вычислительных систем ФГБОУ ВО ПНИПУ (614990, г. Пермь, Комсомольский пр., д. 29, e-mail: d.gaynutdinova@mail.ru). 


\begin{abstract}
About the author
Dinara F. Gaynutdinova (Perm, Russian Federation) - Postgraduate Student, Department of Mechanics of Composite Materials and Structures, Engineer, High-Performance Computer System Centre, Perm National Research Polytechnic University (29, Komsomolsky av., Perm, 614990, Russian Federation, e-mail: d.gaynutdinova@mail.ru).
\end{abstract}

Получено 30.09.2016 